B-03-2020

\title{
BALANCE DE CARBONO DE LAS ZONAS REGABLES DEL TRASVASE TAJO-SEGURA
}

\author{
Martin-Gorriz, B. ${ }^{(1)},(P)$, Gallego-Elvira, B. ${ }^{(1)}$, Martínez-Alvarez, V. ${ }^{(1)}$, Maestre-Valero J.F. ${ }^{(1)}$
}

${ }^{1}$ Escuela Técnica Superior de Ingeniería Agronómica, Universidad Politécnica de Cartagena. Paseo Alfonso XIII, 48. 30203 Cartagena, España. E-mail: b.martin@upct.es

\section{Resumen}

La demarcación hidrográfica del Segura se caracteriza por presentar un importante déficit estructural de agua, que afecta fundamentalmente al regadío, ya que se trata del uso con mayor demanda y se considera de menor prioridad que el suministro a poblaciones. La incorporación de Agua Marina Desalinizada (AMD) es una de las principales estrategias para hacer frente al persistente déficit hídrico que afecta al regadío del sureste español. Con el fin de mejorar el conocimiento sobre la sostenibilidad de la agricultura que se desarrolla en las Zonas Regables del Trasvase Tajo-Segura, en este trabajo se estima la huella de carbono de estos regadíos, para tres escenarios de incremento de AMD. Se realiza el balance de carbono como diferencia entre las emisiones de Gases de Efecto Invernadero (GEI) de las actividades agrícolas, y la remoción de $\mathrm{CO}_{2}$ asociada a los cultivos implantados. Los resultados globales indican que los regadíos del trasvase Tajo-Segura (TTS) representan un importante sumidero de carbono en cualquiera de los escenarios analizados, disminuyendo su capacidad como sumidero de carbono conforme se intensifica la energía específica del suministro de agua de

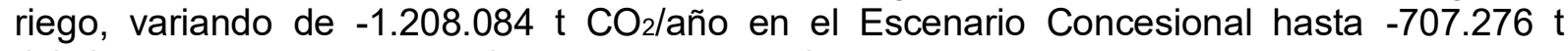

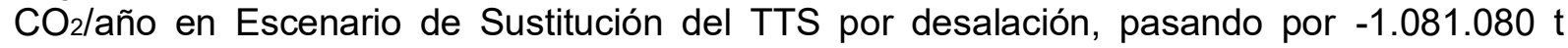

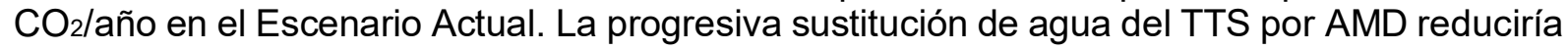
en un $30 \%$ la capacidad de ser sumidero de $\mathrm{CO}_{2}$ de la agricultura. Los resultados por cultivos muestran que todos los cultivos son sumideros de $\mathrm{CO}_{2}$, siendo los leñosos 9 veces más eficientes que los hortícolas. Las UDAs en las que predominan los cultivos hortícolas serían las más perjudicadas por la sustitución de TTS por AMD.

\section{Abstract}

The Segura river basin is characterized by a structural water deficit, which fundamentally affects irrigation, since it is the use with the highest demand and considered to be of lower priority than the urban supply. The use of desalinated sea water (DSW) is one of the main strategies to deal with the persistent water deficit that affects irrigated agriculture in south-east Spain. In order to improve the knowledge about the-sustainability of crops in the Tajo-Segura Irrigated area, this study evaluates the carbon footprint (CF) of the main crops in the area for three scenarios with increasing DSW. The CF was obtained as difference between the greenhouse gas emissions (GHG) of agricultural activities, and the $\mathrm{CO}_{2}$ fixed by crops. Overall, the results show that Tajo-Segura irrigation area represents an important carbon sink in all scenarios, and that the carbon sink capacity is reduced when the specific energy of the irrigation water supply intensifies, varying from $-1,208,084 \mathrm{t} \mathrm{CO}_{2} /$ year in the Concessional Scenario up to $-707,276 \mathrm{t} \mathrm{CO}_{2} /$ year in the TTS water substitution for DSW Scenario, going through $-1,081,080 \mathrm{t} \mathrm{CO}_{2}$ /year in the Current Scenario. The progressive replacement of TTS water by DSW could potentially reduce $\mathrm{CO}_{2}$ sink capacity of agriculture by $30 \%$. The results show that all crops are $\mathrm{CO}_{2}$ sinks, but the woody crops are 9 times more efficient than horticultural crops. Therefore, the UDAs in which horticultural crops dominate are expected to be affected the most by the replacement of TTS water by DSW. 


\section{1.- Introducción}

La agricultura es necesaria para la producción de alimentos, y debe ser sostenible medioambientalmente para que pueda perdurar en el tiempo (CEC, 1999; UN-DSD 2000). La sostenibilidad de un sistema de producción agrícola debe sustentarse en indicadores adecuados. El Ministerio de Agricultura Pesca y Alimentación en su Informe Anual de Indicadores (MAPA, 2018) considera las emisiones de Gases de Efecto Invernadero (GEI) como un indicador adecuado para dar una visión integradora de la sostenibilidad del sector agrario. Desde la perspectiva de las emisiones de Gases de Efecto Invernadero (GEI), los cultivos agrícolas se caracterizan por presentar una doble vertiente. Por un lado, emiten GEI como consecuencia de las labores e insumos que se aportan y, por otra parte, representan un sumidero que captura y almacena el $\mathrm{CO}_{2}$ atmosférico como parte de la fotosíntesis. En consecuencia, para analizar la "huella de carbono" en los cultivos agrícolas es necesario realizar un "balance de carbono" entre las emisiones de GEI y la remoción de $\mathrm{CO}_{2}$. Dependiendo de las técnicas de producción e insumos utilizados se puede obtener un balance positivo, lo que implica que la actividad capta más $\mathrm{CO}_{2}$ del que emite, y consecuentemente la misma se puede considerar sostenible desde esta perspectiva. Por otra parte, un balance negativo implicaría la necesidad de un replanteamiento de las técnicas e insumos productivos para alcanzar la sostenibilidad.

La demarcación hidrográfica del Segura se caracteriza por presentar un importante déficit estructural de agua, que afecta fundamentalmente al regadío, ya que se trata del uso con mayor demanda y se considera de menor prioridad que el suministro a poblaciones (CHS, 2015). A pesar de que en los últimos ciclos de planificación se han desarrollado distintas políticas orientadas a disminuir este déficit hídrico, su magnitud apenas ha variado como consecuencia del impacto del cambio climático en los recursos hídricos convencionales disponibles. La reutilización de aguas regeneradas y la incorporación de Agua Marina Desalinizada (AMD) son las principales estrategias recogidas en la planificación hídrica española con el fin de hacer frente al persistente déficit hídrico que afecta al regadío del sureste español. La principal ventaja del suministro de AMD es su condición de recurso hídrico inagotable y no sujeto a variaciones climáticas; pero el principal inconveniente es el elevado consumo energético asociado a su producción, lo que genera un coste del agua producto muy elevado y un nivel de emisiones de GEI que dificulta el desarrollo de las políticas demandadas para el control del cambio climático.

Con el fin de mejorar el conocimiento sobre la sostenibilidad de la agricultura que se desarrolla en las Zonas Regables del Trasvase Tajo-Segura, en este trabajo se estima de forma analítica y objetiva la huella de carbono de estos regadíos, cuantificando su balance de carbono como diferencia entre las emisiones de GEI de las actividades agrícolas desarrolladas, y la remoción de $\mathrm{CO}_{2}$ asociada a los cultivos implantados.

\section{2.- Objetivos}

La huella de carbono se ha calculado bajo tres escenarios de suministro hídrico en los que se produce una sustitución progresiva del agua procedente del Trasvase Tajo-Segura (TTS) por suministro de agua marina desalinizada (AMD); dado que es la principal estrategia recogida en la planificación hídrica española con el fin de hacer frente al persistente déficit hídrico que afecta al regadío del sureste español. La principal ventaja del suministro de AMD es su condición de recurso hídrico inagotable y no sujeto a variaciones climáticas; pero el principal inconveniente es el elevado consumo energético asociado a su producción, lo que genera un coste del agua producto muy elevado y un nivel de emisiones de GEl que dificulta el desarrollo de las políticas demandadas para el control del cambio climático. 


\section{3.- Materiales y Métodos}

Este trabajo se ha desarrollado en dos fases: (1) determinación de las emisiones y remociones de $\mathrm{CO}_{2}$ eq de los nueve cultivos mayoritarios en las zonas regables asociadas el TTS (alcachofa, brócoli, lechuga, melón, limonero, naranjo, mandarino, albaricoquero y melocotonero), y (2) extrapolación de los valores obtenidos en la fase anterior a los regadíos asociados al TTS. Para determinar las emisiones de GEI de los cultivos se ha aplicado la metodología de Análisis de Ciclo de Vida (ACV), siguiendo los protocolos estandarizados por las normas ISO 14040/14044, como procedimiento de cálculo con mayor reconocimiento y difusión a nivel nacional e internacional. Estas emisiones se han estimado utilizado fuentes de datos oficiales (Ministerios, Confederaciones, Administración Autonómica, etc.) y, en ausencia de estos, recurriendo a la información recogida en los trabajos de otros autores que consideramos de reconocido prestigio. Los datos de remoción de $\mathrm{CO}_{2}$ de los cultivos analizados se han obtenido del "Estudio sobre la absorción de $\mathrm{CO}_{2}$ por los cultivos más representativos de la Región de Murcia" (Victoria, 2010). Este estudio fue realizado por los centros oficiales de investigación y las universidades públicas de la Región (CEBAS-Consejo Superior de Investigaciones Científicas, Instituto Murciano de Investigación y Desarrollo Agrario y Alimentario, Universidad de Murcia y Universidad Politécnica de Cartagena) y analiza el potencial de remoción de $\mathrm{CO}_{2}$ de las principales especies agrícolas y forestales de la Región de Murcia. Para la extrapolación de los valores obtenidos en la primera fase a los regadíos asociados al TTS se han utilizado Sistemas de Información Geográfica (SIG), adoptando como unidad territorial de cálculo las Unidades de Demanda Agraria (UDAs) definidas en el Plan Hidrológico de la Demarcación del Segura para el periodo 2015-2021 (CHS, 2015). Ambas fases del trabajo se han desarrollado bajo tres escenarios de origen del agua de riego: (a) Escenario Concesional según el Plan Hidrológico de la Demarcación del Segura 2015/21 para las Zonas Regables del Trasvase Tajo-Segura; (b) Escenario Actual, ajustando el suministro del trasvase para riego a su valor medio para el periodo 1979-2011 y el suministro de AMD a las concesiones actuales; y (c) Escenario de Sustitución de TTS por desalación, que resulta directamente comparable con el Escenario Concesional, ya que implica el mismo volumen total de suministro.

\section{Justificación de los escenarios considerados}

La actividad del riego es la principal consumidora de energía en la producción de cultivos de regadío bajo las condiciones agroclimáticas de la cuenca del Segura, siendo el origen del agua de riego un factor clave en la energía específica $\left(\mathrm{kWh} / \mathrm{m}^{3}\right)$ asociada a esta actividad (Soto-García et al., 2013). Dado que el factor de emisión de la energía eléctrica en España es significativo $(0,354 \mathrm{~kg} \mathrm{CO} 2$ eq $/ \mathrm{kWh}$, según la base de datos ECOINVENT, en agosto de 2018) y que hay una gran diferencia de energía específica en el suministro desde el TTS $\left(1,21 \mathrm{kWh} / \mathrm{m}^{3}\right.$, Melgarejo y Montaño 2009) y desde las plantas desaladoras de agua marina $\left(4,32 \mathrm{kWh} / \mathrm{m}^{3}\right.$, Martínez-Álvarez et al., 2019), se ha considerado que el origen del agua suministrada es un factor especialmente relevante a la hora de definir distintos escenarios en este estudio (Tabla 1).

\section{$\underline{\text { Justificación de los cultivos considerados }}$}

La estimación de las emisiones de GEI para un cultivo es una actividad que requiere conocer toda la información sobre las labores de cultivo e insumos aportados, así como de las infraestructuras necesarias en parcela. La estimación de la remoción de $\mathrm{CO}_{2}$ requiere de la toma de muestras de individuos vegetales completos para determinar el contenido de carbono de todos sus órganos. Por tanto, no es fácil disponer de toda esta información para la multitud de cultivos que se desarrollan en las Zonas Regables del TTS, pero sí suele está accesible para los más relevantes. Por este motivo, se han seleccionado los 9 cultivos que más superficie ocupan en la Región de Murcia en 2017 (CREM, 2019), como marco geográfico 
más próximo a la cuenca del Segura, de la que no se dispone de información específica por cultivos.

Los cultivos se han organizados en tres grupos: hortícolas al aire libre, cítricos y frutales no cítricos. Esta organización tiene como finalidad ajustarse a la clasificación de cultivos que el Plan Hidrológico de la Demarcación del Segura 2015/21 sigue para la descripción de las UDAs.

Tabla 1. Volumen de agua por recurso para cada escenario.

\begin{tabular}{llcc}
\hline Escenario & Recurso & $\begin{array}{c}\text { Volumen de agua } \\
\text { (hmªño) }\end{array}$ & $\begin{array}{c}\text { Porcentaje } \\
\mathbf{( \% )}\end{array}$ \\
\hline Concesional & Aguas superficiales & 107,53 & 15,89 \\
& Aguas subterráneas & 127,80 & 18,89 \\
& Aguas regeneradas & 41,33 & 6,11 \\
& Suministro del TTS & 400 & 59,11 \\
& Suministro de AMD & 0 & 0 \\
\hline Actual & Aguas superficiales & 107,53 & 19,71 \\
& Aguas subterráneas & 127,80 & 23,42 \\
& Aguas regeneradas & 41,33 & 7,57 \\
& Suministro del TTS & 176 & 32,26 \\
& Suministro de AMD & 93 & 17,04 \\
\hline Concesional & Aguas superficiales & 107,53 & 15,89 \\
& Aguas subterráneas & 127,8 & 18,89 \\
& Aguas regeneradas & 41,33 & 6,11 \\
& Suministro del TTS & 0 & 0 \\
& Suministro de AMD & 400 & 59,11 \\
\hline
\end{tabular}

TTS: Trasvase Tajo-Segura; AMD: Agua Marina Desalinizada.

\section{Emisiones de gases de efecto invernadero de los cultivos}

Para determinar las emisiones de GEI se ha utilizado la metodología de ACV, que tiene en cuenta los procesos de forma global y permite estudiar las cargas ambientales y los impactos potenciales a lo largo del ciclo de vida de un producto o de una actividad.

El ACV es un proceso objetivo que permite evaluar las cargas ambientales asociadas a un producto o cultivo agrícola, identificando y cuantificando el uso de materia y energía y los vertidos al entorno, con el fin de determinar el impacto que ese uso de recursos y esos vertidos producen al medio ambiente. Además, permite evaluar y llevar a la práctica estrategias de mejora ambiental, así como brindar soporte para la toma de decisiones más rentables, sostenibles y eficientes. La complejidad del ACV requiere un protocolo al cual deberá ajustarse todo estudio de ACV. Dicho protocolo se encuentra establecido en la normativa elaborada por International Standards Organisation, ISO 14040 (2006) e ISO 14044 (2006), que conlleva cuatro fases: objetivos y alcance del estudio, análisis del inventario, análisis del impacto e interpretación.

Mediante el ACV se han cuantificado las emisiones directas e indirectas de GEI producidos por los siguientes cultivos hortícolas (alcachofa, brócoli, lechuga y melón) y leñosos (naranjo, mandarino, limonero, albaricoquero y melocotonero) producidos en Zonas Regables del TTS bajo diferentes escenarios de suministro de agua. La unidad funcional utilizada ha sido la hectárea de superficie cultivada y el análisis se ha realizado entre la cuna y la puerta de la parcela (from "cradle to gate"), por lo que se han estudiado las fases de extracción de materias primas, transporte a la explotación, utilización en el cultivo y transporte de residuos (plásticos, etc.) al gestor autorizado correspondiente. 
Las emisiones de GEI se han estimado siguiendo la metodología de Panel Internacional de Cambio Climático (IPCC, 2006), v 1.03 (para un horizonte cronológico de 100 años) que cuantifica las emisiones de GEI referidas a dióxido de carbono equivalente $\left(\mathrm{CO}_{2}\right.$ eq). El desarrollo completo de la metodología, que no ha sido expuesto en el presente documento por limitaciones de espacio, puede consultarse en Martin-Gorriz et al. (2020).

\section{4.- Resultados y Discusión}

Los resultados por grupos de cultivos (Fig. 1) ponen de manifiesto que los cultivos leñosos ("cítricos" y "frutales no cítricos") presentan un balance de carbono por hectárea mucho más favorable que los cultivos "hortícolas al aire libre". Las emisiones de GEI de los "hortícolas al aire libre" son superiores a la de los grupos de cultivos leñosos, ("cítricos" y "frutales no cítricos"), mientras que su remoción de $\mathrm{CO}_{2}$ es sensiblemente inferior, resultando en balances de carbono menos favorables. Respecto a los escenarios, conforme se intensifica la energía específica $\left(\mathrm{kWh} / \mathrm{m}^{3}\right)$ del suministro de agua de riego (Escenario Concesional $\rightarrow$ Escenario Actual $\rightarrow$ Escenario de Sustitución del TTS por desalación) las emisiones de GEI se incrementan, haciendo menos favorable el balance de carbono de cada grupo de cultivos, ya que la remoción de $\mathrm{CO}_{2}$ es independiente del origen del agua de riego. Cabe destacar que en el caso del grupo de cultivos más desfavorable ("hortícolas al aire libre") el balance para el Escenario de Sustitución del TTS por desalación llega a producir valores positivos, lo que implica un flujo global de carbono de la actividad a la atmósfera de 4,52 t CO2/ha-año.

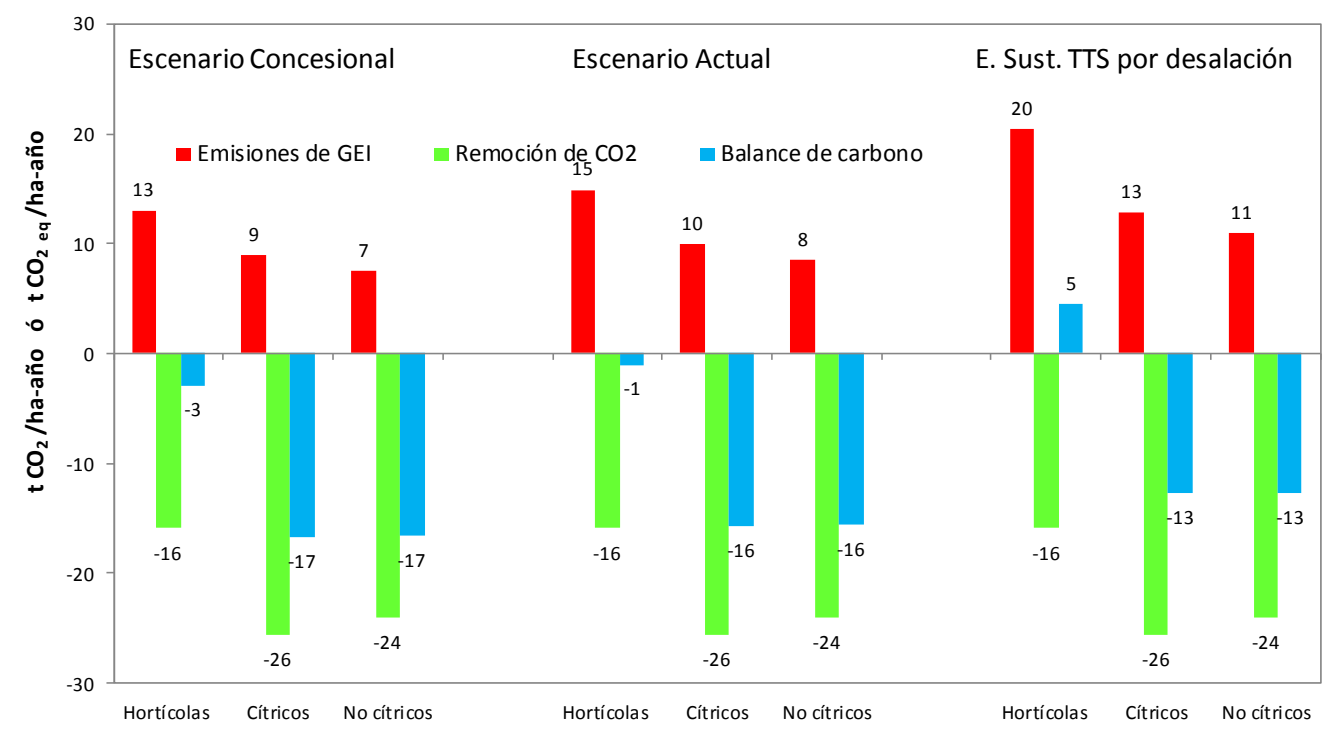

Figura 1. Resultados de emisiones de GEI, remoción de $\mathrm{CO}_{2}$ y balance de carbono por grupos de cultivo y escenarios.

Los resultados globales de emisiones de $\mathrm{GEI}$, remoción de $\mathrm{CO}_{2}$ y balance de carbono para la superficie neta de regadío asociada al trasvase Tajo-Segura en el Plan Hidrológico de la Demarcación del Segura 2015/21 (98.923,6 ha) se muestran en la Fig. 2. Se pone de manifiesto que los regadíos del trasvase Tajo-Segura representan un importante sumidero de carbono en cualquiera de los escenarios, disminuyendo su capacidad como sumidero de

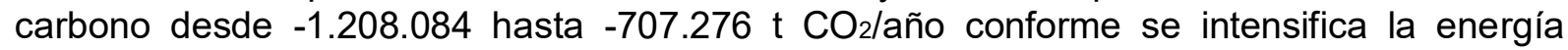
específica $\left(\mathrm{kWh} / \mathrm{m}^{3}\right)$ del suministro de agua de riego (Escenario Concesional $\rightarrow$ Escenario Actual $\rightarrow$ Escenario de Sustitución del TTS por desalación). La remoción de $\mathrm{CO}_{2}$ no varía

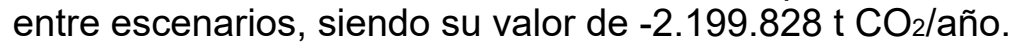




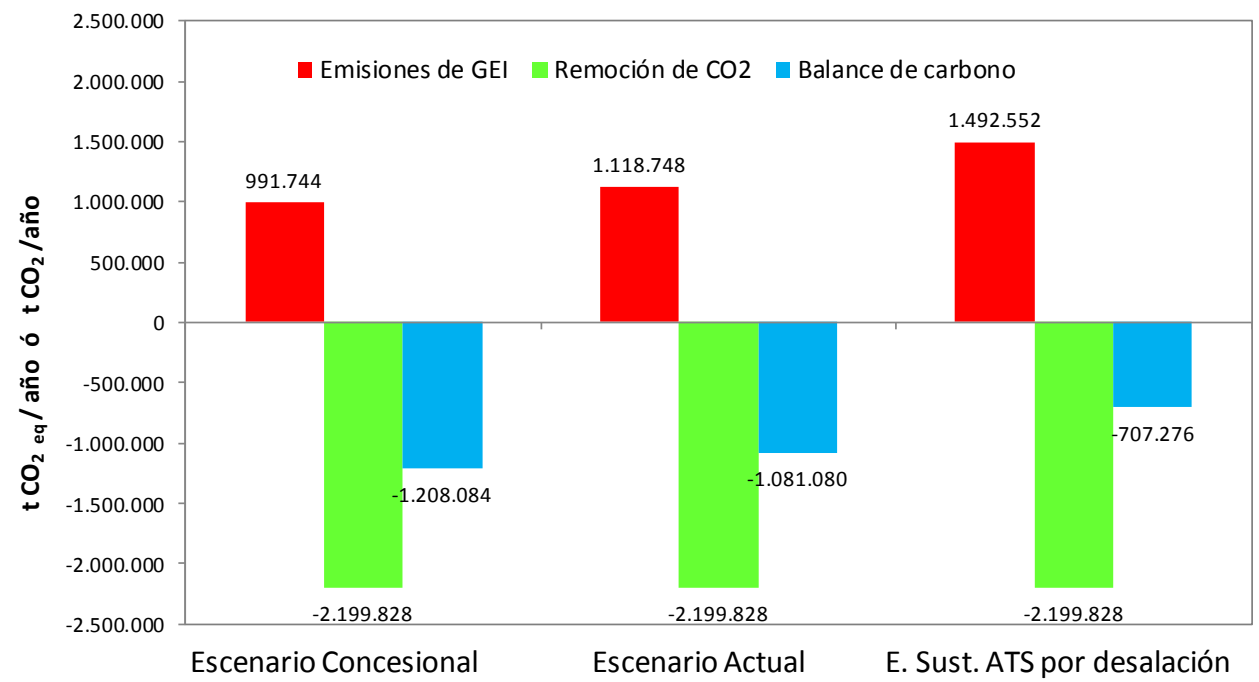

Figura 2. Resultados de emisiones de $\mathrm{GEI}$, remoción de $\mathrm{CO}_{2}$ y balance de carbono para la superficie neta de regadío asociada al trasvase Tajo-Segura en el Plan Hidrológico de la Demarcación del Segura 2015/21(98.923,6 ha), por escenarios.

Sin embargo, el rol como sumidero de los regadíos de trasvase Tajo-Segura podría ser superior si se suministrase el $100 \%$ de las concesiones de las Zonas Regables del trasvase, lo que debería permitir regar la superficie nominal según concesiones de las Zonas Regables del trasvase (133.356 ha, Soto-García et al., 2014). La Fig. 3 recoge los resultados globales de emisiones de $\mathrm{GEI}$, remoción de $\mathrm{CO}_{2}$ y balance de carbono para este caso en el Escenario Concesional y el Escenario de Sustitución del TTS por desalación, que implican la misma cantidad de suministro de agua. Se observa como la capacidad como sumidero de

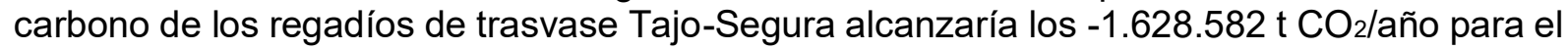

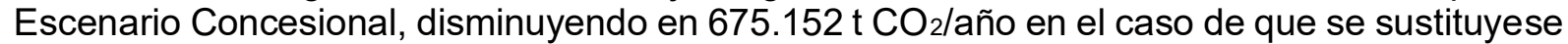
el suministro de $400 \mathrm{hm}$ /año del TTS por agua marina desalinizada (Escenario de Sustitución del TTS por desalación). Para este caso hipotético, la remoción de $\mathrm{CO}_{2}$ alcanzaría -2.965.523 t CO /año en ambos escenarios.

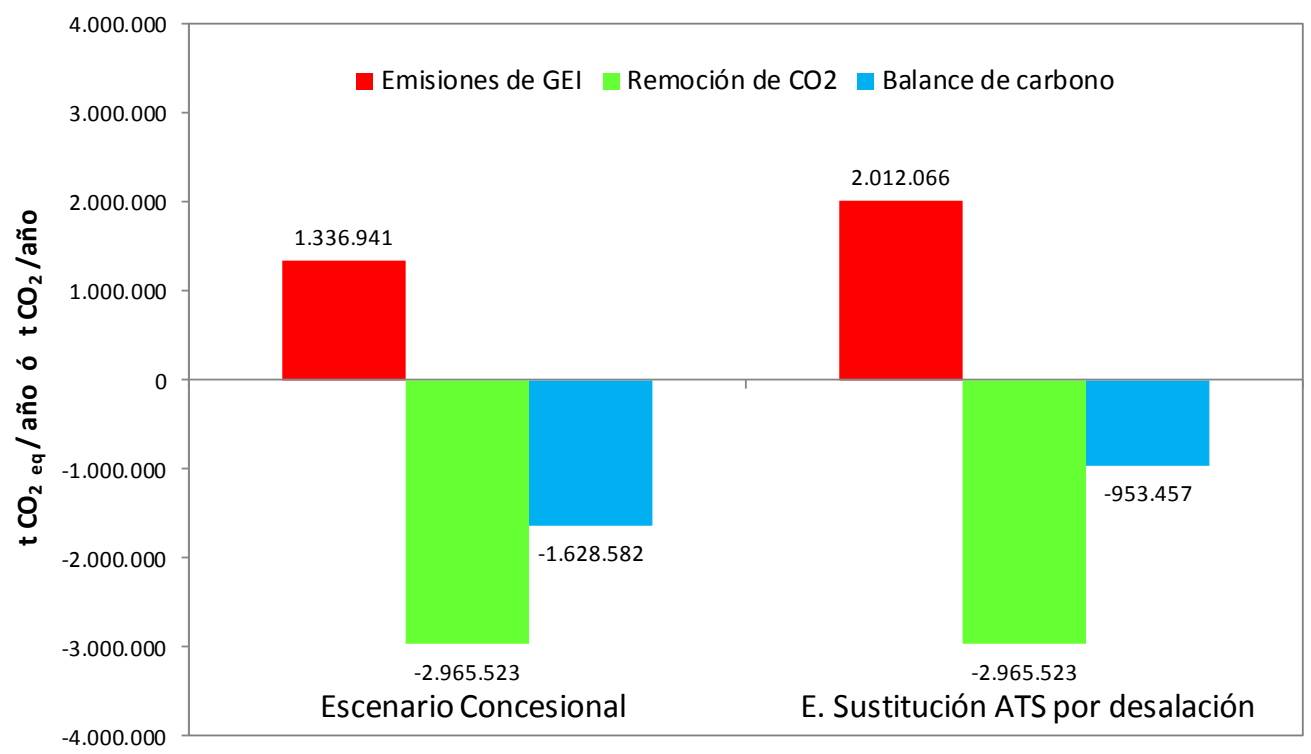

Figura 3. Resultados de emisiones de $\mathrm{GEI}$, remoción de $\mathrm{CO}_{2}$ y balance de carbono para la superficie la superficie nominal según concesiones de las Zonas Regables del trasvase (133.356 ha), por escenarios. 
Finalmente, la Fig.4 muestra para cada escenario los valores medios por hectárea de emisiones de $\mathrm{GEI}$, remoción de $\mathrm{CO}_{2}$ y balance de carbono para los regadíos asociados al TTS. Se observa que la tasa de emisiones de GEI aumenta conforme aumenta la energía específica del suministro hídrico (Escenario Concesional $\rightarrow$ Escenario Actual $\rightarrow$ Escenario de Sustitución del TTS por desalación), mientras que la tasa de remoción de $\mathrm{CO}_{2}$ se mantiene constante en -22,24 t CO$/$ ha-año. Como resultado el balance se va haciendo menos favorable, pasando de -12,21 t CO 2 /ha-año en el Escenario Concesional a -7,14 t CO $/$ ha-año en el Escenario de Sustitución del TTS por desalación. Cabe destacar que el análisis de esta misma información por UDAs pone de manifiesto como aquellas en las que hay un mayor predominio de los cultivos hortícolas al aire libre (UDA 61, UDA 70 y UDA 71) llegan a perder su condición de sumidero de carbono en el Escenario de Sustitución del TTS por desalación.

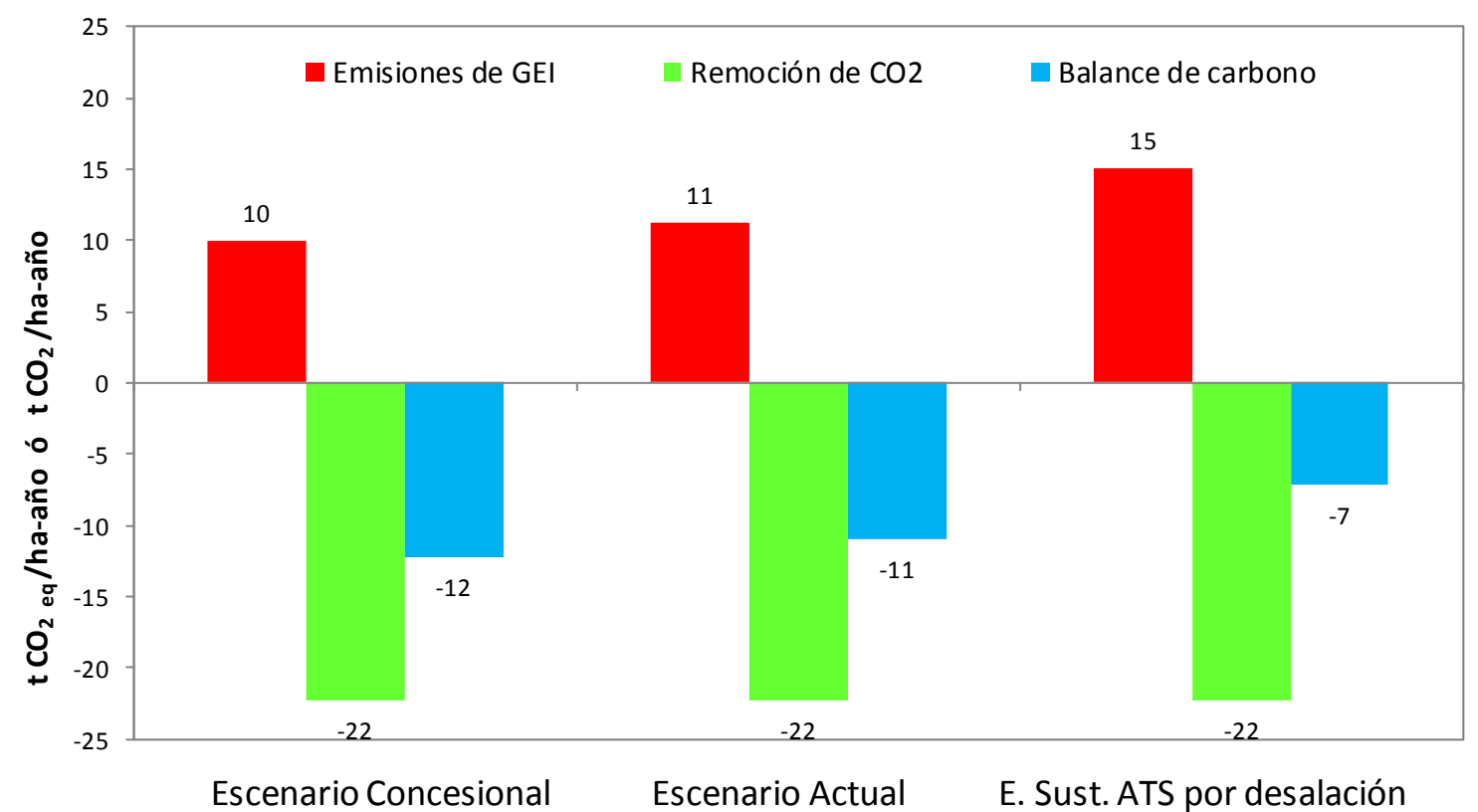

Figura 4. Valores medios por hectárea de emisiones de $\mathrm{GEI}$, remoción de $\mathrm{CO}_{2}$ y balance de carbono para los regadíos asociada al trasvase Tajo-Segura, por escenarios.

\section{5.- Conclusiones}

Las principales conclusiones de este trabajo son:

- Todos los cultivos son sumideros de $\mathrm{CO}_{2}$, siendo los leñosos son 9 veces más eficientes que los hortícolas.

- El riego supone el $43 \%$ de las emisiones de GEI en el escenario actual, y alcanzaría el $57 \%$ en el escenario de sustitución del TTS por desalación.

- Las emisiones de GEI aumentarían un 14\% al pasar del escenario actual al escenario de sustitución del TTS por desalación.

- El riego $100 \%$ con AMD reduciría la capacidad de los cultivos de ser sumideros de $\mathrm{CO}_{2}$ en un $30 \%$ respecto al escenario actual; y un $41 \%$ respecto al escenario concesional.

- Las UDAs en las que predominan los cultivos hortícolas serían las más perjudicadas por la sustitución de TTS por AMD. 


\section{6.- Agradecimientos}

Las distintas actividades necesarias para el desarrollo de este trabajo han sido financiadas por (1) el Ministerio de Economía y Competitividad y el Fondo Europeo de Desarrollo Regional a través del proyecto RIDESOST (AGL2017-85857-C2-2-R), y (2) por la Cátedra Universidad-Empresa "Trasvase y Sostenibilidad - José Manuel Claver Valderas", creada por el Sindicato Central de Regantes del Acueducto Tajo-Segura (SCRATS) y la Universidad Politécnica de Cartagena (UPCT).

\section{7.- Bibliografía}

CEC, Commission of the European Communities, (1999). Directions towards sustainable agriculture. Commission of the European Communities, Bruselas, Países Bajos.

CHS, Confederación Hidrográfica del Segura, (2015). Plan Hidrológico de la Demarcación del Segura https://www.chsegura.es/chs/planificacionydma/planificacion15-21/.

CREM, Centro Regional de Estadística de Murcia (2019). http://econet.carm.es/inicio//crem/sicrem/PU datosBasicos/sec49.html

IPCC, Intergovernmental Panel on Climate Change (2006). Guidelines for national greenhouse gas inventories. In: HAS, Eggleston, Biennia, L., Miwa, K., Negara, T., Tanabe, K. (Eds.), Prepared by National Greenhouse Gas Inventories Programme. IGES, Japan: Eggleston Published.

ISO, International Organization for Standardization, 14040 (2006). Environmental management - Life cycle assessment - Principles and framework. ISO, Geneva.

ISO, International Organization for Standardization, 14044 (2006). Environmental management - Life cycle assessment - Requirements and Guidelines. ISO, Geneva.

MAPA, Ministerio de Agricultura Pesca y Alimentación (2018). Informe Anual de Indicadores 2017: Agricultura, Pesca, Alimentación y Medio Ambiente. Madrid. 211 pp.

Martin-Gorriz, B., Martínez-Alvarez, V., Maestre-Valero, J.F. \& Gallego Elvira, B. (2020). Balance de carbono de las zonas regables del trasvase Tajo-Segura. Sindicato Central de Regantes del Acueducto Tajo-Segura, Cátedra Universidad-Empresa "Trasvase y Sostenibilidad - José Manuel Claver Valderas" de la Universidad Politécnica de Cartagena, 57 pp. http://www.scrats.es/memorias-e-informes.html

Martínez-Alvarez, V., Maestre-Valero, J.F., González-Ortega, M.J., Gallego Elvira, B. \& Martin-Gorriz, B. (2019). Characterization of the agricultural supply of desalinated seawater in Southeastern Spain. Water 2019, 11, 1233; http://dx.doi.org/10.3390/w11061233.

Melgarejo, J. \& Montaño, B. (2009). La eficiencia energética del trasvase Tajo-Segura. Cuaderno Interdisciplinar de Desarrollo Sostenible, 3, 173-193.

Soto-García, M., Martin-Gorriz, B., García-Bastida, P.A., Alcon, F. \& Martínez-Alvarez, V. (2013). Energy consumption for crop irrigation in a semiarid climate (south-eastern Spain). Energy, 55, 1084-1093. http://dx.doi.org/10.1016/j.energy.2013.03.034

Soto-García, M., Martínez-Alvarez, V. \& Martín-Górriz, B. (2014). El regadío en la Región de Murcia. Caracterización y análisis mediante indicadores de gestión. Murcia, 267 pp.

UN-DSD, United Nations Division for Sustainable Development (2000). Agenda 21. Chapter 14: Promoting sustainable agriculture and rural development. United Nations (UN), Nueva York, Estados Unidos.

Victoria, F. (2010). Etiquetado de carbono en las explotaciones y productos agrícolas. La iniciativa murciana como sumidero de $\mathrm{CO}_{2}$. CARM, Murcia, pp. 39-62. http://www.lessco2.es/documentacion.htm 